\title{
Effects of the coadministration of Huatuo Zaizao pills and aspirin on hemorrheology and blood coagulation in rabbits
}

\author{
SHUJUN QU* , TIAN WANG ${ }^{*}$, JIANQIAO ZHANG, XUE SUN, PENGFEI YU, LIANG KONG, \\ YATING CHONG, XIAOLEI QIU and FENGHUA FU
}

\author{
Department of Pharmacology, School of Pharmacy, Yantai University, Yantai, Shandong 264005, P.R. China
}

Received December 20, 2012; Accepted February 26, 2013

DOI: 10.3892/br.2013.76

\begin{abstract}
Huatuo Zaizao pills (HT) is a compound used in Chinese medicine for the treatment of cerebrovascular diseases. The present study aimed to investigate the effects of the coadministration of HT and aspirin on hemorrheology and blood coagulation in rabbits. Rabbits were randomly divided into the control, HT, aspirin and HT plus aspirin groups ( $n=5$ animals per group). The rabbits were treated with HT at a dose of $1 \mathrm{~g} / \mathrm{kg}$, aspirin at a dose of $5 \mathrm{mg} / \mathrm{kg}$ or HT $(1 \mathrm{~g} /$ $\mathrm{kg})$ plus aspirin $(5 \mathrm{mg} / \mathrm{kg})$ administered by gavage. Blood samples were collected prior to treatment and at 2 or $4 \mathrm{~h}$ after treatment. Compared to the control group, there were no significant changes in whole blood viscosity (WBV), plasma viscosity (PV), activated partial thromboplastin time (APTT), prothrombin time (PT), thrombin time (TT) and fibrinogen content (FIB) in the HT or the aspirin group. Coadministration of HT and aspirin prolonged APTT, PT and TT and decreased WBV and PV. The findings indicated that coadministration of HT and aspirin exerted a more potent effect on hemorrheology and blood coagulation, compared to each agent administered alone, and may be a novel approach to the prevention and treatment of hemorrheological disorders.
\end{abstract}

\section{Introduction}

Stroke remains a major healthcare problem, with a significant human and economic toll. According to the World Health Organization, 15 million people suffer from stroke worldwide each year. Of these, 5 million succumb to the stroke and a further 5 million become permanently disabled (1). Primary and secondary prevention plays a significant role in reducing the burden of stroke. Aspirin remains the cornerstone of

Correspondence to: Professor Fenghua Fu, Department of Pharmacology, School of Pharmacy, Yantai University, 30 Qingquan Road, Yantai, Shandong 264005, P.R. China

E-mail: fufenghua@sohu.com

*Contributed equally

Key words: coadministration, Huatuo Zaizao pills, aspirin, blood coagulation, hemorrheology primary and secondary stroke prevention (2) and is increasingly being used in the prevention and treatment of ischemic stroke. However, it may also be accompanied with severe gastrointestinal complications, including bleeding, perforation and ulceration. Whereas a lower dose of aspirin may reduce its risks, it may also be accompanied by lower effectiveness. Furthermore, an increasing number of reports on aspirin resistance has led to a growing concern among clinicians and patients, regarding the efficacy of aspirin treatment. Based on a recent meta-analysis in vitro or laboratory studies, aspirin resistance is a factor independent of future vascular events (3). It was reported that aspirin fails to inhibit platelet aggregation in 5-55\% of individuals and this is an important cause of clinical aspirin resistance or failure $(4,5)$.

Hemorrheological disorders play an important role in the pathogenesis and development of several diseases, such as ischemic stroke, coronary artery disease and diabetic complications. It was reported that promoting blood circulation to relieve blood stasis may improve hemorrheological events. Traditional Chinese medicine (TCM), which is mostly based on formulations, has attracted increasing attention as a complementary therapeutic method to western medicine (6).

Huatuo Zaizao pills (HT), a pure natural preparation derived from plants, is a TCM formulation marketed in China. In 1985, it was formally listed in the key scientific and technical projects during the sixth Five-Year Plan by the National Commission for Science and Technology. Over the past few years, studies that are only partly published in the Chinese medical literature, on the pharmacognosy, pharmaceutics and pharmacology of HT, have been conducted by 14 medical research institutions. HT is widely used in the treatment and prevention of cerebrovascular diseases, apoplexy and hemiplegia. The aim of the present study was to evaluate the effects of the coadministration of HT and aspirin on hemorrheology and blood coagulation in rabbits.

\section{Materials and methods}

Animals. New Zealand rabbits, weighing 2.5-3.0 kg (provided by the Experimental Animal Center of Shandong Engineering Research Center for Natural Drugs), were housed in conventional cages with free access to food and water, at a temperature of $22.0 \pm 2.0^{\circ} \mathrm{C}$ and a relative humidity of $50 \pm 5 \%$, with a $12-\mathrm{h}$ light-dark cycle. Prior to the experiment, the rabbits were 
Table I. Effects of the coadministration of Huatuo Zaizao pills (HT) and aspirin on whole blood viscosity (WBV) at low shear rate.

\begin{tabular}{lccc}
\hline & \multicolumn{3}{c}{ WBV $\left(\mathrm{mPa} . \mathrm{s}, 10 \mathrm{sec}^{-1}\right)$} \\
\cline { 2 - 4 } Groups & $0 \mathrm{~h}$ & $2 \mathrm{~h}$ & $4 \mathrm{~h}$ \\
\hline Control & $7.24 \pm 1.04$ & $7.49 \pm 0.94$ & $7.51 \pm 0.71$ \\
HT & $7.45 \pm 0.66$ & $7.31 \pm 0.87$ & $6.75 \pm 0.62$ \\
Aspirin & $7.01 \pm 0.91$ & $7.07 \pm 0.60$ & $6.75 \pm 0.52$ \\
HT + aspirin & $7.19 \pm 0.78$ & $7.32 \pm 0.92$ & $5.90 \pm 0.59^{\mathrm{a}, \mathrm{b}}$ \\
\hline
\end{tabular}

Data are expressed as the means \pm SEM $(n=5)$. One-way ANOVA was used to analyze the significant differences among the different groups The comparison between two groups was performed with the Student's unpaired t-test. Compared to control, ${ }^{\text {a }} \mathrm{P}<0.05$. Compared to prior to treatment, ${ }^{\mathrm{b}} \mathrm{P}<0.05$.

Table II. Effects of the coadministration of Huatuo Zaizao pills (HT) and aspirin on whole blood viscosity (WBV) at high shear rate.

\begin{tabular}{lccc}
\hline & \multicolumn{3}{c}{ WBV $\left(\mathrm{mPa} . \mathrm{s}, 150 \mathrm{sec}^{-1}\right)$} \\
\cline { 2 - 4 } Groups & $0 \mathrm{~h}$ & $2 \mathrm{~h}$ & $4 \mathrm{~h}$ \\
\hline Control & $3.61 \pm 0.82$ & $3.70 \pm 0.56$ & $3.60 \pm 0.47$ \\
HT & $3.61 \pm 0.33$ & $3.75 \pm 0.47$ & $3.41 \pm 0.71$ \\
Aspirin & $3.51 \pm 0.29$ & $3.47 \pm 0.10$ & $3.50 \pm 0.18$ \\
HT + aspirin & $3.53 \pm 0.26$ & $2.92 \pm 0.30^{\mathrm{a}, \mathrm{b}}$ & $2.99 \pm 0.34^{\mathrm{a}, \mathrm{b}}$ \\
\hline
\end{tabular}

Data are expressed as the means \pm SEM $(n=5)$. One-way ANOVA was used to analyze the significant differences among the different groups The comparison between two groups was performed with the Student's unpaired t-test. Compared to control, ${ }^{\text {a }}<<0.05$. Compared to prior to treatment, ${ }^{\mathrm{b}} \mathrm{P}<0.05$.

allowed to acclimatize for 1 week. All animal experiments were conducted with the approval of the Yantai University Committee for the Care and Use of Laboratory Animals.

Drugs and chemicals. HT were purchased from Guangzhou Qixing Pharmaceutical Co., Ltd. (Guangzhou, China). Aspirin was obtained from Bayer HealthCare Manufacturing S.r.1. (Beijing, China). Urethane was obtained from Sinopharm Chemical Reagent Co., Ltd. (Shanghai, China).

Experimental design. Twenty New Zealand rabbits were randomly assigned to 4 groups ( $\mathrm{n}=5$ rabbits per group): control group rabbits were treated with distilled water $(1 \mathrm{ml} / \mathrm{kg})$ by gavage; HT group rabbits were treated with $\mathrm{HT}$ at a dose of $1 \mathrm{~g} /$ $\mathrm{kg}$ by gavage; aspirin group rabbits were treated with aspirin at a dose of $5 \mathrm{mg} / \mathrm{kg}$ by gavage; HT plus aspirin group rabbits were treated with HT at a dose of $1 \mathrm{~g} / \mathrm{kg}$, plus aspirin at a dose of $5 \mathrm{mg} / \mathrm{kg}$ by gavage. Blood samples were collected from the carotid aortas prior to treatment and at 2 or $4 \mathrm{~h}$ after treatment. Blood was collected into plastic tubes with $3.8 \%$ sodium citrate (citrate:blood=1:9, v/v) for the evaluation of whole blood viscosity (WBV). Plasma was then separated from blood by centrifugation at $3,000 \mathrm{xg}$ for $10 \mathrm{~min}$ for the detection of plasma viscosity (PV) and plasma anticoagulation.

Viscosity determination. A total of $800 \mu \mathrm{l}$ blood or plasma was used to determine the viscosity with a blood viscometer
(LBY-N6COMPACT; Beijing Precil Instrument Co., Ltd, Beijing, China) at $37^{\circ} \mathrm{C} . \mathrm{WBV}$ was measured at the shear rates of 10 and $150 \mathrm{sec}^{-1}$. PV was measured at the shear rate of $150 \mathrm{sec}^{-1}$.

Plasma anticoagulation assay. Activated partial thromboplastin time (APTT), prothrombin time (PT), thrombin time (TT) and fibrinogen content (FIB) were assessed with commercial kits following the manufacturer's instructions with a coagulometer (NC-050; Rayto Life and Analytical Sciences Co., Ltd., Shenzhen, China). APTT was determined by incubating $50 \mu \mathrm{l}$ of plasma with $50 \mu \mathrm{l}$ of APTT-activating agent for $3 \mathrm{~min}$ at $37^{\circ} \mathrm{C}$, followed by the addition of $50 \mu \mathrm{l} \mathrm{CaCl}{ }_{2}$. PT was determined by incubating $50 \mu \mathrm{l}$ of plasma solution for $3 \mathrm{~min}$ at $37^{\circ} \mathrm{C}$, followed by the addition of $100 \mu \mathrm{l}$ of thromboplastin agent. TT was determined by incubating $50 \mu 1$ of plasma solution for $3 \mathrm{~min}$ at $37^{\circ} \mathrm{C}$, followed by the addition of $100 \mu \mathrm{l}$ of thrombin agent. FIB was determined by incubating $10 \mu \mathrm{l}$ of plasma with $90 \mu \mathrm{l}$ of imidazole buffer for $3 \mathrm{~min}$ at $37^{\circ} \mathrm{C}$, followed by the addition of $50 \mu \mathrm{l}$ of FIB agent.

Data analysis. Data were analyzed using SPSS 17.0 statistical software and are expressed as the means \pm SEM. One-way ANOVA was used to analyze the significant differences among the different groups. Comparisons between two groups were performed with the Student's unpaired t-test. $\mathrm{P}<0.05$ was considered to indicate a statistically significant difference between groups. 
Table III. Effects of the coadministration of Huatuo Zaizao pills (HT) and aspirin on plasma viscosity (PV).

\begin{tabular}{lccc}
\hline & \multicolumn{2}{c}{$\mathrm{PV}\left(\mathrm{mPa} . \mathrm{s}, 150 \mathrm{sec}^{-1}\right)$} \\
\cline { 2 - 4 } Groups & $0 \mathrm{~h}$ & $2 \mathrm{~h}$ & $4 \mathrm{~h}$ \\
\hline Control & $1.35 \pm 0.23$ & $1.51 \pm 0.39$ & $1.46 \pm 0.22$ \\
HT & $1.28 \pm 0.08$ & $1.46 \pm 0.21$ & $1.36 \pm 0.16$ \\
Aspirin & $1.40 \pm 0.28$ & $1.36 \pm 0.19$ & $1.32 \pm 0.22$ \\
HT + aspirin & $1.31 \pm 0.25$ & $1.11 \pm 0.05$ & $0.91 \pm 0.16^{\mathrm{a}, \mathrm{b}}$
\end{tabular}

Data are expressed as the means \pm SEM $(n=5)$. One-way ANOVA was used to analyze the significant differences among the different groups. The comparison between two groups was performed with the Student's unpaired t-test. Compared to control, ${ }^{a} \mathrm{P}<0.05$. Compared to prior to treatment, ${ }^{\mathrm{b}} \mathrm{P}<0.05$.

Table IV. Effects of the coadministration of Huatuo Zaizao pills (HT) and aspirin on activated partial thromboplastin time (APTT).

\begin{tabular}{lccc}
\hline & \multicolumn{2}{c}{ APTT (sec) } \\
\cline { 2 - 4 } Groups & $0 \mathrm{~h}$ & $2 \mathrm{~h}$ & $4 \mathrm{~h}$ \\
\hline Control & $15.00 \pm 2.33$ & $13.74 \pm 2.56$ & $14.24 \pm 2.38$ \\
HT & $14.24 \pm 2.64$ & $13.20 \pm 2.49$ & $14.58 \pm 2.84$ \\
Aspirin & $14.86 \pm 1.83$ & $13.24 \pm 2.19$ & $12.82 \pm 2.40$ \\
HT + aspirin & $14.22 \pm 1.38$ & $14.54 \pm 2.06$ & $18.26 \pm 2.45^{\mathrm{a}, \mathrm{b}}$ \\
\hline
\end{tabular}

Data are expressed as the means \pm SEM $(n=5)$. One-way ANOVA was used to analyze the significant differences among the different groups The comparison between two groups was performed with the Student's unpaired t-test. Compared to control, ${ }^{\mathrm{a}} \mathrm{P}<0.05$. Compared to prior to treatment, ${ }^{\mathrm{b}} \mathrm{P}<0.05$.

\section{Results}

Effects of the coadministration of $H T$ and aspirin on $W B V$. The effects of the coadministration of HT and aspirin on WBV are presented in Tables I and II. Compared to the control group, treatment with HT or aspirin alone exerted no effects on WBV. At $4 \mathrm{~h}$ following coadministration of HT and aspirin, the WBV of the HT plus aspirin group was significantly decreased at low shear rate $(\mathrm{P}<0.05)$. At 2 and $4 \mathrm{~h}$ following coadministration of HT and aspirin, the WBV of the HT plus aspirin group was significantly decreased at high shear rate $(\mathrm{P}<0.05)$.

Effects of the coadministration of HT and aspirin on PV. At 2 and $4 \mathrm{~h}$ following treatment with HT or aspirin alone, there were no significant differences in PV in the HT or the aspirin group, compared to that in the control group $(\mathrm{P}>0.05)$. Compared to the control group or prior to treatment, coadministration of $\mathrm{HT}$ and aspirin significantly reduced $\mathrm{PV}$ at the shear rate of $150 \mathrm{sec}^{-1}(\mathrm{P}<0.05$, Table III).

Effects of the coadministration of HT and aspirin on plasma coagulation parameters. As shown in Tables IV-VII, at $4 \mathrm{~h}$ following the coadministration of HT and aspirin, APTT, PT and TT were significantly increased when compared to those in the control group $(\mathrm{P}<0.05)$. Compared to the control group, treatment with HT or aspirin alone exerted no effects on plasma coagulation parameters $(\mathrm{P}>0.05)$. Furthermore, there were no significant differences in FIB content among the 4 groups.

\section{Discussion}

Hemorrheology studies blood flow in relation to pressure, flow volume and resistance in blood vessels, as well as WBV, PV and the disorders of blood coagulation parameters (APTT, PT, TT and FIB level). Several prospective trials confirmed that the increased WBV is a major risk factor for ischemic stroke and heart disease $(7,8)$ and the rheologic changes may affect the clinical outcome of patients with cerebral or coronary arterial diseases $(9,10)$. Recent data also demonstrated that $>80 \%$ of strokes are ischemic and the main factors underlying the pathomechanism of brain ischemia are arterial obstruction and microcirculatory stasis. Previous studies reported a significantly elevated PV, erythrocyte aggregation and impaired red blood cell deformability in ischemic stroke patients (11). Data obtained from several studies confirmed that all ischemic strokes are associated with significant rheological abnormalities, independent of the subtype $(12,13)$. It is possible that in patients with acute ischemic stroke, increased WBV leads to a further reduction of cerebral vascular flow in regions of already compromised flow (14). Therefore, hemorrheological parameters may be important in the development of brain ischemia. 
Table V. Effects of the coadministration of Huatuo Zaizao pills (HT) and aspirin on prothrombin time (PT).

\begin{tabular}{lccc}
\hline & \multicolumn{3}{c}{ PT (sec) } \\
\cline { 2 - 4 } Groups & $0 \mathrm{~h}$ & $2 \mathrm{~h}$ & $4 \mathrm{~h}$ \\
\hline Control & $7.40 \pm 0.50$ & $7.12 \pm 1.00$ & $7.18 \pm 0.44$ \\
HT & $7.70 \pm 1.23$ & $7.26 \pm 1.33$ & $7.82 \pm 1.43$ \\
Aspirin & $7.46 \pm 1.35$ & $7.28 \pm 1.37$ & $7.72 \pm 1.45$ \\
HT + aspirin & $7.68 \pm 1.05$ & $8.06 \pm 1.77$ & $9.66 \pm 1.55^{\mathrm{a}, \mathrm{b}}$
\end{tabular}

Data are expressed as the means \pm SEM $(n=5)$. One-way ANOVA was used to analyze the significant differences among the different groups. The comparison between two groups was performed with the Student's unpaired t-test. Compared to control, ${ }^{a} \mathrm{P}<0.05$. Compared to prior to treatment, ${ }^{\mathrm{b}} \mathrm{P}<0.05$.

Table VI. Effects of the coadministration of Huatuo Zaizao pills (HT) and aspirin on thrombin time (TT).

\begin{tabular}{lrrr}
\hline & \multicolumn{3}{c}{ TT (sec) } \\
\cline { 2 - 4 } Groups & \multicolumn{1}{c}{$0 \mathrm{~h}$} & $2 \mathrm{~h}$ & $4 \mathrm{~h}$ \\
\hline Control & $14.12 \pm 1.54$ & $14.60 \pm 2.08$ & $14.94 \pm 2.48$ \\
HT & $14.3 \pm 1.94$ & $14.16 \pm 2.33$ & $16.40 \pm 2.81$ \\
Aspirin & $13.32 \pm 0.86$ & $14.84 \pm 1.93$ & $13.20 \pm 1.29$ \\
HT + aspirin & $13.82 \pm 1.36$ & $15.20 \pm 1.74$ & $18.52 \pm 2.17^{\mathrm{a}, \mathrm{b}}$
\end{tabular}

Data are expressed as the means \pm SEM $(n=5)$. One-way ANOVA was used to analyze the significant differences among the different groups. The comparison between two groups was performed with the Student's unpaired t-test. Compared to control, ${ }^{\text {a }}<0.05$. Compared to prior to treatment, ${ }^{\mathrm{b}} \mathrm{P}<0.05$.

Table VII. Effects of the coadministration of Huatuo Zaizao pills (HT) and aspirin on fibrinogen content (FIB).

\begin{tabular}{lccr}
\hline & & \multicolumn{2}{c}{ FIB $(\mathrm{g} / \mathrm{l})$} \\
\cline { 2 - 4 } Groups & $0 \mathrm{~h}$ & $2 \mathrm{~h}$ & $4 \mathrm{~h}$ \\
\hline Control & $3.30 \pm 0.46$ & $3.32 \pm 0.42$ & $3.40 \pm 0.36$ \\
HT & $3.20 \pm 0.34$ & $2.94 \pm 0.28$ & $3.02 \pm 0.34$ \\
Aspirin & $3.34 \pm 0.54$ & $3.20 \pm 0.46$ & $3.00 \pm 0.30$ \\
HT + aspirin & $3.32 \pm 0.47$ & $3.26 \pm 0.54$ & $2.98 \pm 0.46$ \\
\hline
\end{tabular}

Data are expressed as the means \pm SEM $(n=5)$. One-way ANOVA was used to analyze the significant differences among the different groups The comparison between two groups was performed with the Student's unpaired t-test.

WBV is an important parameter in hemorrheology, which reflects the rheological properties of blood and blood cells. The major determinants of WBV are PV, hematocrit and the properties of red blood cells, including erythrocyte deformability and erythrocyte aggregation (15). WBV is a reflection of the intrinsic resistance of blood to flow in vessels. WBV abnormalities have been associated with increased risk of ischemic stroke and the reduced blood fluidity may expedite tissue ischemia in atherosclerotic diseases (14). PV is a factor that significantly influences the blood perfusion velocity through the capillaries. The viscosity of capillary blood is closely related to $\mathrm{PV}$, together with all the consequences of blood perfusion in the microcirculation (16). Lower capillary blood viscosity results in higher blood perfusion velocity in the macrocirculation. In the present study, treatment with HT or aspirin alone exerted no effects on WBV or PV. However, at $4 \mathrm{~h}$ following the coadministration of HT and aspirin, WBV and PV were significantly decreased. The results demonstrated that HT and aspirin may have a synergetic effect on decreasing WBV and PV.

Under normal physiological conditions, a balance exists between factors that promote and those that retard coagulation. Coagulation disorders may lead to an increased risk of clotting. PT and APTT are performance indicators reflecting the effi- 
cacy of both the intrinsic and extrinsic coagulation pathways Shortening of PT and APTT reflects an increased tendency to clot (17). In our study, the coadministration of HT and aspirin significantly prolonged APTT, PT and TT. However, treatment with HT or aspirin alone exerted no effects on plasma coagulation parameters. The results indicated that the synergistic effect of HT and aspirin on WBV and PV may be correlated with both extrinsic and intrinsic coagulation systems.

In conclusion, our study has demonstrated that treatment with HT or low-dose aspirin alone exerted no effects on hemorrheology and blood coagulation. However, the coadministration of HT and aspirin prolonged APTT, PT and TT and decreased WBV and PV. The findings indicated that this coadministration exerted a more potent effect on hemorrheology and blood coagulation, compared to each agent administered individually, and may be a novel approach to the prevention and treatment of hemorrheological disorders.

\section{Acknowledgements}

This study was supported by the National Natural Science Foundation of China (no. 81274125), the Project of Fundamental Study Funds of the Shandong Provincial Education Department (no. J11LF56) and the Doctoral Foundation of Yantai University (no. YX12B30).

\section{References}

1. Caro JJ, Huybrechts KF and Duchesne I: Management patterns and costs of acute ischemic stroke: an international study. For the Stroke Economic Analysis Group. Stroke 31: 582-590, 2000.

2. Apostolakis S, Marin F and Lip GY: Antiplatelet therapy in stroke prevention. Adv Cardiol 47: 141-154, 2012.
3. Pusch G, Feher G, Kotai K, et al: Aspirin resistance: focus on clinical endpoints. J Cardiovasc Pharmacol 52: 475-484, 2008.

4. Mirkhel A, Peyster E, Sundeen J, et al: Frequency of aspirin resistance in a community hospital. Am J Cardiol 98: 577-579, 2006.

5. Uchiyama S, Nakamura T, Yamazaki M, Kimura Y and Iwata M New modalities and aspects of antiplatelet therapy for stroke prevention. Cerebrovasc Dis 21 (Suppl 1): S7-S16, 2006

6. Normile D: Asian medicine. The new face of traditional Chinese medicine. Science 299: 188-190, 2003.

7. Lee AJ, Mowbray PI, Lowe GD, Rumley A, Fowkes FG and Allan PL: Blood viscosity and elevated carotid intima-media thickness in men and women: the Edinburgh Artery Study. Circulation 97: 1467-1473, 1998.

8. Suarez C, Castillo J, Suarez P, Naveiro J and Lema M: The prognostic value of analytical hemorheological factors in stroke. Rev Neurol 24: 190-192, 1996 (In Spanish).

9. Velcheva I and Nikolova G: Hemorheological disturbances and cognitive function in patients with cerebrovascular disease. Clin Hemorheol Microcirc 39: 397-402, 2008.

10. Kesmarky G, Kenyeres P, Rabai M and Toth K: Plasma viscosity: a forgotten variable. Clin Hemorheol Microcirc 39: 243-246, 2008.

11. Ernst E, Matrai A and Marshall M: Blood rheology in patients with transient ischemic attacks. Stroke 19: 634-636, 1988

12. Fisher M and Meiselman HJ: Hemorheological factors in cerebral ischemia. Stroke 22: 1164-1169, 1991.

13. Wood JH and Kee DB Jr: Hemorheology of the cerebral circulation in stroke. Stroke 16: 765-772, 1985.

14. Nemeth N, Soukup J, Menzel M, et al: Local and systemic hemorheological effects of cerebral hyper- and hypoperfusion in a porcine model. Clin Hemorheol Microcirc 35: 59-65, 2006.

15. Dutta A and Tarbell JM: Influence of non-Newtonian behavior of blood on flow in an elastic artery model. J Biomech Eng 118: 111-119, 1996.

16. Marossy A, Svorc P, Kron I and Gresova S: Hemorheology and circulation. Clin Hemorheol Microcirc 42: 239-258, 2009.

17. Chan KC, Yin MC and Chao WJ: Effect of diallyl trisulfide-rich garlic oil on blood coagulation and plasma activity of anticoagulation factors in rats. Food Chem Toxicol 45: 502-507, 2007. 